\title{
LETTERS
}

\section{Extracorporeal lung assist might avoid invasive ventilation in exacerbation of COPD}

\section{To the Editors:}

Acute exacerbation of chronic obstructive pulmonary disease (COPD) requiring invasive mechanical ventilation (IMV) is associated with a very poor prognosis. Therefore noninvasive mechanical ventilation (NIV) with avoidance of endotracheal intubation should be preferred. However, NIV can fail and result in severe respiratory acidosis. If IMV becomes mandatory, the development of pulmonary dynamic hyperinflation with subsequent barotrauma and circulatory failure is the main pathophysiological alteration.

Extracorporeal $\mathrm{CO}_{2}$ elimination, such as that provided by an arteriovenous extracorporeal lung assist system (avECLA), combined with NIV might be a therapeutic option in order to avoid IMV in patients with acute exacerbation of COPD (AECOPD). We present three cases with hypercapnic respiratory failure that were treated with NIV and extracorporeal lung assist for 2, 7 and 8 days, respectively. The combination of these two therapeutic approaches might be effective to prevent the potentially lethal side-effects of IMV in this group of multimorbid patients.

COPD affects $4-7 \%$ of the general population and is the fourth leading cause of death in developed countries [1]. If patients with AECOPD require mechanical ventilation (MV), the mortality rate is $17-30 \%$ [2].

MV is mandatory in patients with AECOPD if decompensated hypercapnic respiratory failure is evident. NIV via face mask or helmet has been the evidence-based treatment of choice in AECOPD patients with hypercapnia for more than 20 yrs [3]. NIV has been shown to improve gas exchange and outcome when compared with IMV. However, in some cases NIV fails to eliminate $\mathrm{CO}_{2}$ sufficiently and tracheal intubation and IMV are traditionally regarded as the last treatment option [4]. The prognosis of the affected patients still remains very poor and, therefore, measures that result in avoidance of IMV might be rational [5].

Over $10 \mathrm{yrs}$ ago, a pumpless avECLA consisting of an extracorporeal membrane lung with a minimal resistance to blood flow, that was shown to remove $\mathrm{CO}_{2}$ highly effectively in patients with acute respiratory distress syndrome, was introduced into clinical practice. AvECLA enables minute ventilation and peak inspiratory pressures to be reduced significantly following the rules of lung protective ventilation [6]. This was also shown for patients with asthma treated with IMV [7].

Thus, in AECOPD patients with decompensated respiratory acidosis that is refractory to NIV, the addition of avECLA might be able to avoid tracheal intubation and subsequent IMV. We report three AECOPD patients with decompensated respiratory acidosis treated with avECLA plus NIV.

The institutional review board approval to publish this patient data was obtained from the ethics committee of the University of Würzburg (Würzburg, Germany).

After ultrasonographic examination of the targeted vessels and exclusion of severe peripheral artery disease (PAD), avECLA was implanted using a 13-F cannula for the femoral artery and a 15- or $17-F$ cannula for the femoral vein, respectively. Anticoagulation was performed with a continuous heparin infusion resulting in a partial thromboplastin time of 40-60 s. With membrane lung blood flows ranging from 1.2 to $1.5 \mathrm{~L} \cdot \mathrm{min}^{-1}$ and oxygen sweep gas flows through the membrane lung of $1-10 \mathrm{~L} \cdot \mathrm{min}^{-1}$, the arterial carbon dioxide tension $\left(\mathrm{Pa}_{1} \mathrm{CO}_{2}\right)$ was adjusted to values between 50 and $65 \mathrm{mmHg}$ resulting in a $\mathrm{pH}$ of $>7.2$ (table 1 ). NIV was continued during avECLA treatment and positive endexpiratory pressure and pressure support were titrated to avoid tachypnoea and maintain oxygenation at an arterial oxygen tension $\left(\mathrm{Pa}_{2} \mathrm{O}_{2}\right)$ between 50 and $60 \mathrm{mmHg}$.

An 85-yr-old morbidly obese male patient was admitted to the emergency room and subsequently to the intensive care unit (ICU) with shortness of breath. His past medical history (PMH) consisted of congestive heart failure and COPD with ambulant oxygen treatment for $8 \mathrm{~h} \cdot \mathrm{day}^{-1}$. The chest radiograph and thoracic computed tomography (CT) scan showed cardiomegaly, basal pneumonic infiltrates and a known left indurative pleurisy. Assuming AECOPD, a calculated antibiotic therapy was initiated and piperacillin/tazobactam and prednisolone were administered. The patient developed decompensated respiratory acidosis. NIV via face mask did not result in amelioration of hypercapnia. $4 \mathrm{~h}$ after admission to the ICU avECLA was implanted. NIV via helmet combined with avECLA was performed for 8 days. Respiratory mechanics improved and signs of infection resolved. On day eight avECLA was removed uneventfully, NIV was continued for four more days without further decompensation of the respiratory acidosis. The patient was adapted to a home continuous positive airway pressure system and discharged home 4 days later.

A 66-yr-old male patient was admitted to the hospital with incremental deterioration of a chronic pulmonary infection. His main complaints were shortness of breath and continuously increasing adynamia. His PMH consisted of congestive heart failure, ventricular tachycardia with subsequent implantation of an automated cardioverter and COPD. The chest radiograph and the thoracic CT scan showed cardiomegaly, bilateral pneumonic infiltrates and right-sided bonchiectasis. Assuming AECOPD, a calculated antibiotic therapy was initiated and piperacillin/ tazobactam and prednisolone were administered. The patient 
TABLE 1 Patient gas exchange, ventilator settings, haemodynamics and extracorporeal lung parameters

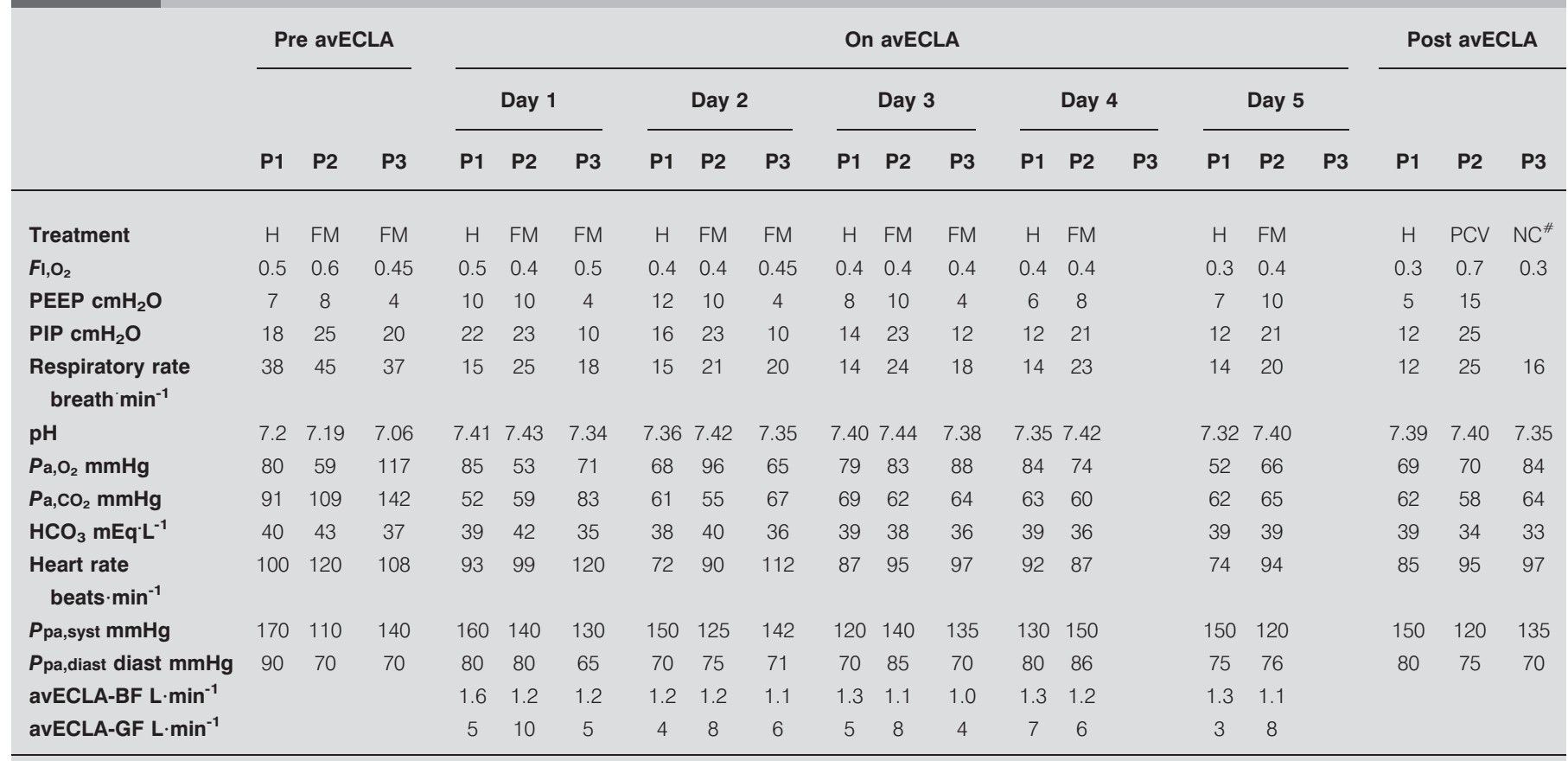

avECLA: arteriovenous extracorporeal lung assist; P: patient; $\mathrm{H}$ : helmet; FM: face mask; PCV: pressure controlled ventilation; $\mathrm{NC}$ : nasal cannula; Fl, $\mathrm{O}_{2}$ : inspiratory oxygen fraction; PEEP: positive end-expiratory pressure; PIP: peak inspiratory pressure; $\mathrm{Pa}_{1} \mathrm{O}_{2}$ : arterial oxygen tension; $\mathrm{Pa}_{1} \mathrm{CO}_{2}$ : arterial carbon dioxide tension; $\mathrm{HCO}_{3}$ : bicarbonate level; Ppa,syst: systolic pulmonary artery pressure; Ppa,diast: diastolic pulmonary artery pressure; avECLA-BF: avECLA blood flow; avECLA-GF: avECLA gas flow. ${ }^{\#}$ : gas flow via nasal cannula $=2 \mathrm{O}_{2} \mathrm{~L} \cdot \mathrm{min}^{-1}$

developed decompensated respiratory acidosis despite NIV via face mask. $12 \mathrm{~h}$ after admission to the ICU avECLA was implanted. NIV via face mask combined with avECLA was performed for 7 days. However, respiratory mechanics did not improve and after an initial phase of amelioration, oxygenation and haemodynamics deteriorated further $\left(\mathrm{Pa}_{\mathrm{a}} \mathrm{O}_{2} \quad 40 \mathrm{mmHg}\right.$, respiratory rate 35 breaths $\cdot \mathrm{min}^{-1}$ and need for vasopressors) and signs of infection increased due to recurrence of pneumonia. Antibiotic therapy was switched to ceftazidim/ciprofloxacin. The patient's trachea was intubated and IMV in a lung protective mode was performed. AvECLA was discontinued uneventfully. Tracheostomy was performed on the same day. On day 17 the patient was transferred to a weaning clinic and subsequently to a nursing home providing long-term respiratory care with IMV.

A 52-yr-old cachectic female patient was admitted to the ICU in severe respiratory distress. Auscultation of the chest revealed wheezing and a prolonged expiration. Her $\mathrm{PMH}$ was remarkable for arterial hypertension, COPD and pneumococcus pneumonia infection requiring IMV that was accompanied by acute renal failure 1 yr ago. NIV via face mask and anti-asthmatic medication were started immediately but did not ameliorate the severe respiratory acidosis with a $\mathrm{Pa}_{1} \mathrm{CO}_{2}$ of $140 \mathrm{mmHg}$. The chest radiograph showed bilateral infiltrates and the typical radiological signs of COPD. Calculated antibiotic therapy was initiated and ceftriaxone and clarithromycin and a bolus of prednisolone were administered. After avECLA was started a $\mathrm{pH}>7.2$ was quickly achieved. Bronchospasm subsided and avECLA was discontinued 2 days later uneventfully. Nevertheless, due to insufficiency of the respiratory pump the patient was adapted to an ambulatory NIV system and subsequently discharged home.

The longer IMV is performed, the higher the risk of death [8]. Thus, avoidance of IMV is a rational therapeutic goal. In all three patients the combination of avECLA and NIV unloaded the respiratory pump and brought the pulmonary gas exchange to acceptable values (table 1). In two cases IMV was avoided, in one case the concept failed due to recurrence of pneumonia and subsequent weaning failure.

A prerequisite to put the presented treatment approach into practice is the concept of dissociating the pulmonary gas exchange into its two components: $\mathrm{CO}_{2}$ elimination is provided with an extracorporeal lung and oxygenation is secured by an adequate level of mean airway pressure [9]. Whenever hypoxaemia occurs, the presented concept becomes obsolete, since $\mathrm{O}_{2}$ transfer via avECLA does not suffice [6].

Looking at the risk-benefit ratio, complications and costs related to avECLA need to be addressed. It is well known that arterial cannulation with large bore cannulas can cause limb ischaemia especially in multi-morbid patients with a high risk of PAD [6] Without negatively influencing the $\mathrm{CO}_{2}$ elimination rate, this risk can be reduced if cannulas with a diameter of $>15-\mathrm{F}$ are avoided. In addition, looking at the rapid progress in cannula and pump technology development, venovenous extracorporeal $\mathrm{CO}_{2}$ removal with double lumen cannulas requiring only one vascular access port might also be combined with NIV in patients with AECOPD even when PAD is evident [10]. Obviously costs for 
extracorporeal systems must be considered as well as ventilator free days and length of ICU stay.

In conclusion, the combination of NIV and extracorporeal $\mathrm{CO}_{2}$ elimination might be effective to prevent IMV and its potentially lethal side-effects in patients with AECOPD.

Jörg Brederlau*, Thomas Wurmb", Stefan Wilczek*, Kirstin Will*, Sebastian Maier", Markus Kredel ${ }^{\#}$, Norbert Roewer ${ }^{\#}$ and Ralf M. Muellenbach ${ }^{\#}$ *Dept of Intensive Care Medicine, HELIOS Klinikum BerlinBuch, Berlin, "Dept of Anaesthesia and Critical Care, University of Würzburg, and 'Dept of Internal Medicine, University of Würzburg, Würzburg, Germany.

Correspondence: J. Brederlau, Klinik für Intensivmedizin, HELIOS Klinikum Berlin-Buch, Schwanebecker Chaussee 50, 13125 Berlin, Germany. E-mail: joerg.brederlau@helios-kliniken.de

Statement of Interest: A statement of interest for R.M.

Muellenbach can be found at www.erj.ersjournals.com/site/ misc/statements.xhtml

\section{REFERENCES}

1 Ambrosino N, Simonds A. The clinical management in extremely severe COPD. Respir Med 2007; 101: 1613-1624.
2 Berry CE, Wise RA. Mortality in COPD: causes, risk factors, and prevention. COPD 2010; 7: 375-382.

3 Brochard L, Isabey D, Piquet J, et al. Reversal of acute exacerbations of chronic obstructive lung disease by inspiratory assistance with a face mask. N Engl J Med 1990; 323: 1523-1530.

4 Quinnell TG, Pilsworth S, Shneerson JM, et al. Prolonged invasive ventilation following acute ventilatory failure in COPD: weaning results, survival, and the role of noninvasive ventilation. Chest 2006; 129: 133-139.

5 Barnes PJ. New therapies for chronic obstructive pulmonary disease. Med Princ Pract 2010; 19: 330-338.

6 Muellenbach RM, Kredel M, Wunder C, et al. Arteriovenous extracorporeal lung assist as integral part of a multimodal treatment concept: a retrospective analysis of 22 patients with ARDS refractory to standard care. Eur J Anaesthesiol 2008; 25: 897-904.

7 Elliot SC, Paramasivam K, Oram J, et al. Pumpless extracorporeal carbon dioxide removal for life-threatening asthma. Crit Care Med 2007; 35: 945-948.

8 Esteban A, Anzueto A, Frutos F, et al. Characteristics and outcomes in adult patients receiving mechanical ventilation: a 28-day international study. JAMA 2002; 287: 345-355.

9 Gattinoni L, Kolobow T, Tomlinson T, et al. Control of intermittent positive pressure breathing (IPPB) by extracorporeal removal of carbon dioxide. Br J Anaesth 1978; 50: 753-758.

10 Terragni P, Maiolo G, Ranieri VM. Role and potentials of low-flow $\mathrm{CO}_{2}$ removal system in mechanical ventilation. Curr Opin Crit Care 2012; 18: 93-98.

\section{Influence of social deprivation and air pollutants on serious asthma}

\section{To the Editors:}

Asthma is an important disorder worldwide, as a major cause of hospital admissions, medical consultations, prescriptions and impaired quality of life. The precise causes of asthma, although largely unknown, are multifactorial and involve a complex interaction of genetic and environmental factors.

Although hospital admission rates are often increased for people in lower socioeconomic groups [1], little has been reported specifically for serious asthma (i.e. prolonged admissions and/or death within 30 days). Evidence about the effects of air pollutants on the occurrence of serious asthma is also unclear [2].

We aimed to establish the hospital admission rate and case fatality for serious asthma, and whether admissions are associated more strongly with social deprivation or air pollutants.

We used medical record linkage of inpatient data from the Patient Episode Database for Wales (PEDW) and mortality data from the National Health Service (NHS) Welsh Administrative Register. PEDW covers inpatient admissions to all NHS hospitals across 22 local health authorities in Wales, UK (population three million) and has been used as the basis of many previous published studies.
We included all emergency admissions from April 1, 1999 to March 31, 2007 where asthma (ICD-10 codes J45 and J46) was the principal diagnosis at discharge. Patients of all ages with admissions lasting $\geqslant 3$ days, or who died (from any cause) within 30 days, were classified as "serious" cases. We included all first "serious" admissions during the study period, and subsequent serious admissions providing they occurred $>30$ days after the preceding serious admission.

We measured social deprivation and its seven domains using the Welsh Index of Multiple Deprivation 2005 [3]. Social deprivation scores were assigned to 1,896 lower super output areas (LSOAs) across Wales (average LSOA population 1,560) [3]. The LSOAs were ranked according to their social deprivation score and categorised into quintiles (I: least deprived; V: most deprived).

Data on the following eight air pollutants were provided by the AEA Energy and Environment Company (the official British air pollution monitoring agency) from 1999-2007: benzene, carbon monoxide, nitric oxide, nitrogen dioxide, ozone, particulate matter $<10.0 \mu \mathrm{m} \cdot \mathrm{m}^{-3}$ and $<2.5 \mu \mathrm{m} \cdot \mathrm{m}^{-3}$ in diameter and sulfur dioxide [4]. The air pollutants were based on annual means of hourly measurements. In 2007 (2005 for ozone), they were interpolated from 23 sites to geographical levels of $1 \mathrm{~km}^{2}$ [4]. 